\title{
Heterogeneous Formation of Sulfur Species on Manganese Oxides: Effects of Particle Type and Moisture Condition
}

\author{
Tao Wang ${ }^{1}$, Yangyang Liu ${ }^{1}$, Yue Deng ${ }^{1}$, Hanyun Cheng ${ }^{1}$, Xiaozhong Fang ${ }^{1}$, Liwu Zhang ${ }^{1,2 *}$ \\ ${ }^{1}$ Shanghai Key Laboratory of Atmospheric Particle Pollution and Prevention, Department of
}

Environmental Science \& Engineering, Fudan University, Shanghai, 200433, Peoples’ Republic of China

${ }^{2}$ Shanghai Institute of Pollution Control and Ecological Security, Shanghai, 200092, Peoples' Republic of China

Number of Pages: 13

Number of Figures: 7

Number of Tables: 3 
In-situ DRIFTS (diffuse reflectance infrared Fourier transform spectroscopy) is helpful in discussing the species formed on particles. The schematic diagram of the in-situ setup is present in Figure S1. The manganese oxide particles were placed in a ceramic sample cup (0.35 mm depth, $5 \mathrm{~mm}$ i.d.). Mass flow controllers (Beijing Sevenstar electronics Co., LTD) were used to adjust the fluxes of reactant gases to the desired flow rate, concentration and relative humidity $(\mathrm{RH})$. A temperature controller was connected to the DRIFTS chamber (Praying Mantis Kit, Harrick) to control the reaction temperature (298 K).

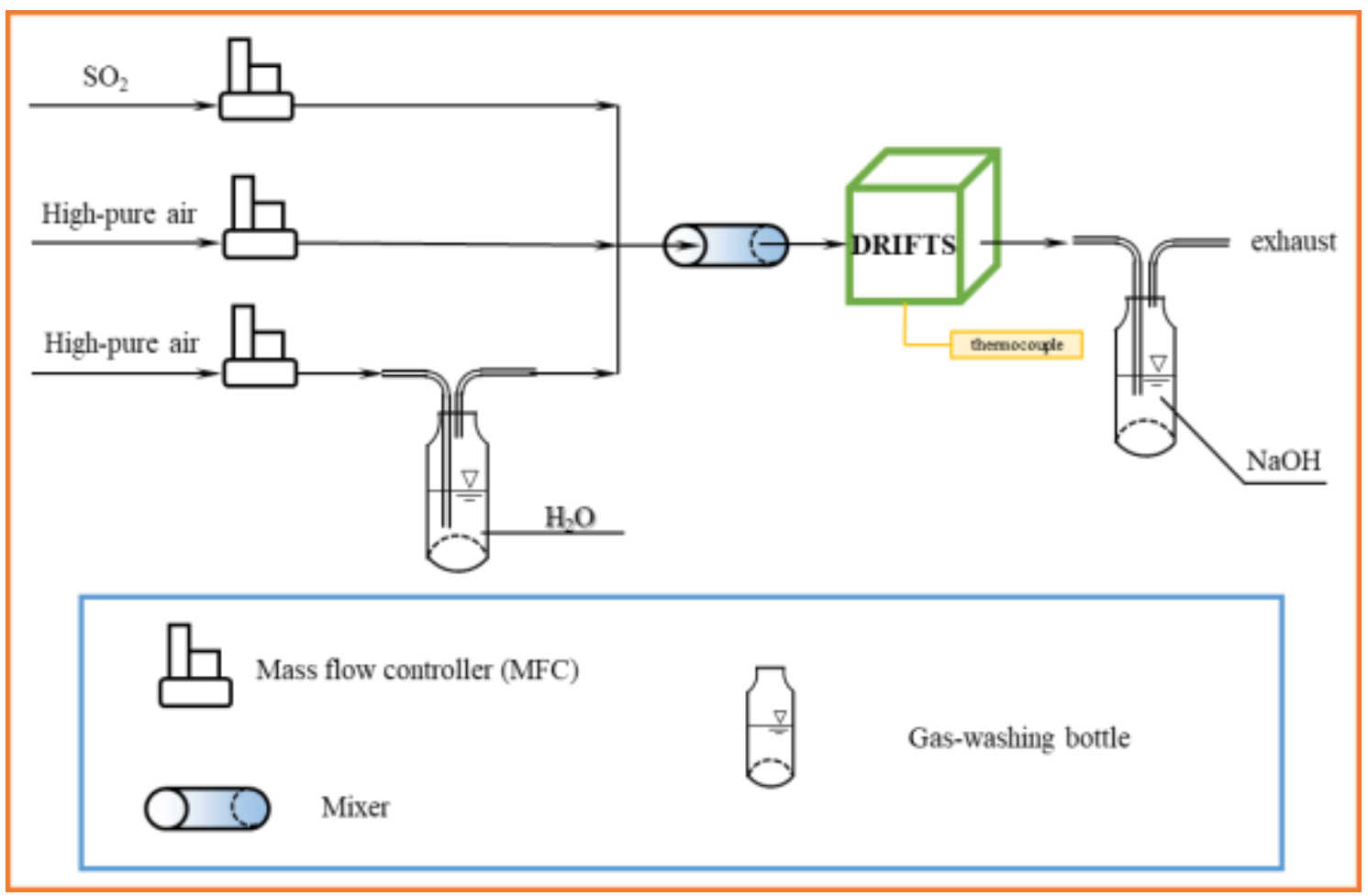

Figure S1. Schematic diagram of experimental setup. The DRIFTS chamber is linked with other parts through Teflon tube. MFC: mass flow controller 
Table S1. Parameters for the uptake coefficient estimations.

\begin{tabular}{|c|c|c|}
\hline \multicolumn{2}{|c|}{ Parameter (unit) } & Value \\
\hline \multicolumn{2}{|c|}{ Sulfate formation rate: $\mathrm{d}\left[\mathrm{SO}_{4}^{2-}\right] / \mathrm{dt}\left(\right.$ ion $\left.\cdot \mathrm{s}^{-1}\right)$} & According to reactions \\
\hline \multirow{2}{*}{\multicolumn{2}{|c|}{ Particle reactive surface area: $\mathrm{As}\left(\mathbf{m}^{2}\right)$}} & $\mathrm{S}_{\mathrm{BET}} \times$ sample mass \\
\hline & & \\
\hline & $\mathrm{A}_{\text {geo }}\left(\mathrm{m}^{2}\right)$ & $1.86 \times 10^{-5}$ (not used in this work) \\
\hline \multirow{4}{*}{\multicolumn{2}{|c|}{ Reactant concentration: $\left[\mathrm{SO}_{2}\right]\left(\right.$ molecule $\left.\cdot \mathrm{cm}^{-3}\right)$}} & $1.52 \times 10^{14}(\mathrm{MnO})$ \\
\hline & & $1.82 \times 10^{14}\left(\mathrm{Mn}_{3} \mathrm{O}_{4}\right)$ \\
\hline & & $3.02 \times 10^{14}\left(\mathrm{Mn}_{2} \mathrm{O}_{3}\right)$ \\
\hline & & $4.50 \times 10^{14}\left(\mathrm{MnO}_{2}\right)$ \\
\hline \multirow{4}{*}{ Velocity of molecule: $v_{\mathrm{SO}_{2}}$} & Gas constant: $\mathrm{R}\left(\mathrm{J} \cdot \mathrm{mol}^{-1} \cdot \mathrm{K}^{-1}\right)$ & 8.314 \\
\hline & Temperature: T (Kelvin) & 298 \\
\hline & Molar mass: $M_{50_{2}}\left(\mathrm{~g} \cdot \mathrm{mol}^{-1}\right)$ & 64.06 \\
\hline & $\mathrm{Pi}: \pi$ (dimensionless) & 3.1416 \\
\hline
\end{tabular}

\section{Page S3}



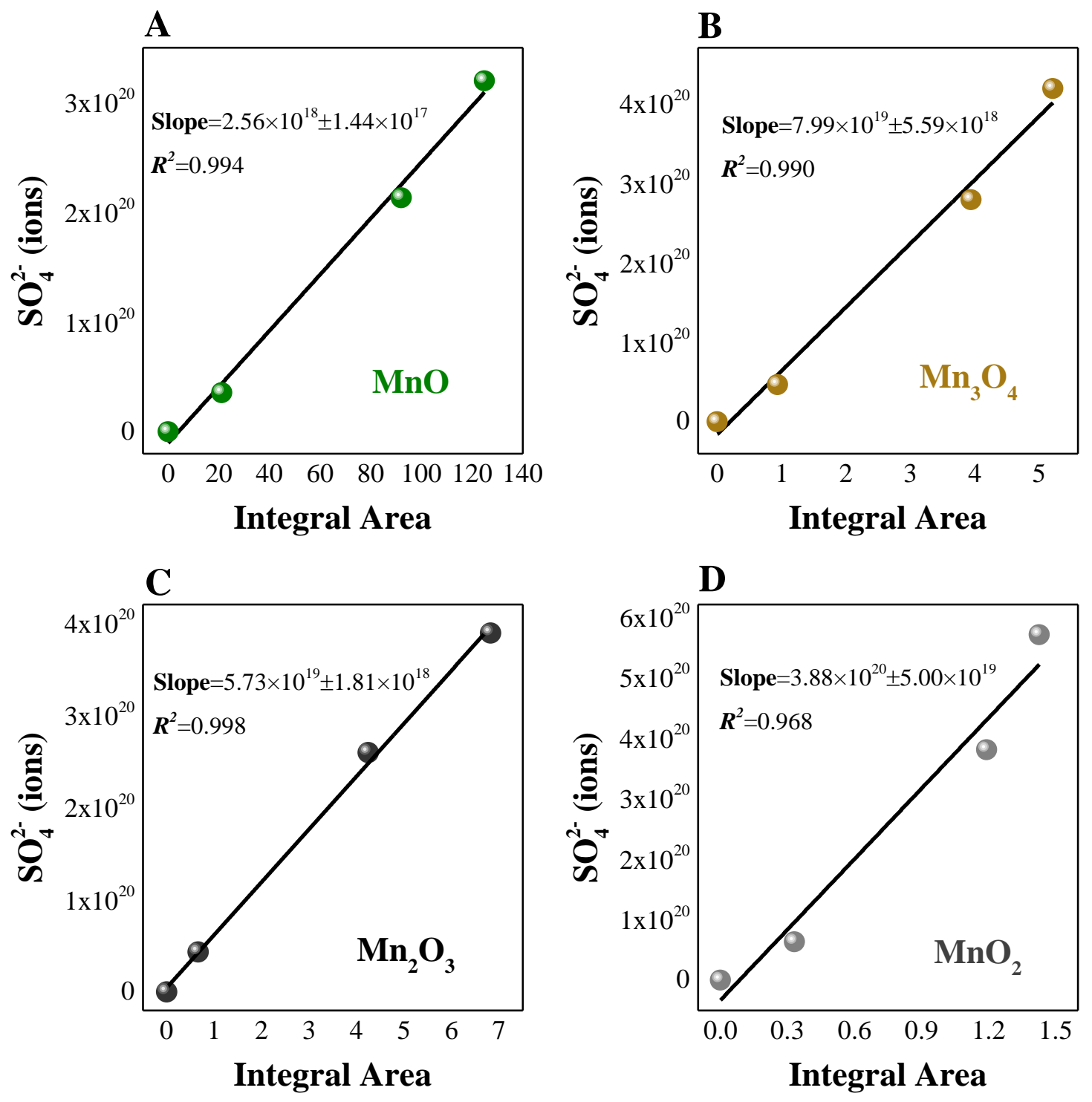

Figure S2. Calibration curves for the integrated areas versus the sulfate concentration. (A: $\mathrm{MnO}, \mathrm{B}: \mathrm{Mn}_{3} \mathrm{O}_{4}$, C: $\mathrm{Mn}_{2} \mathrm{O}_{3}, \mathrm{D}: \mathrm{MnO}_{2}$ ) 


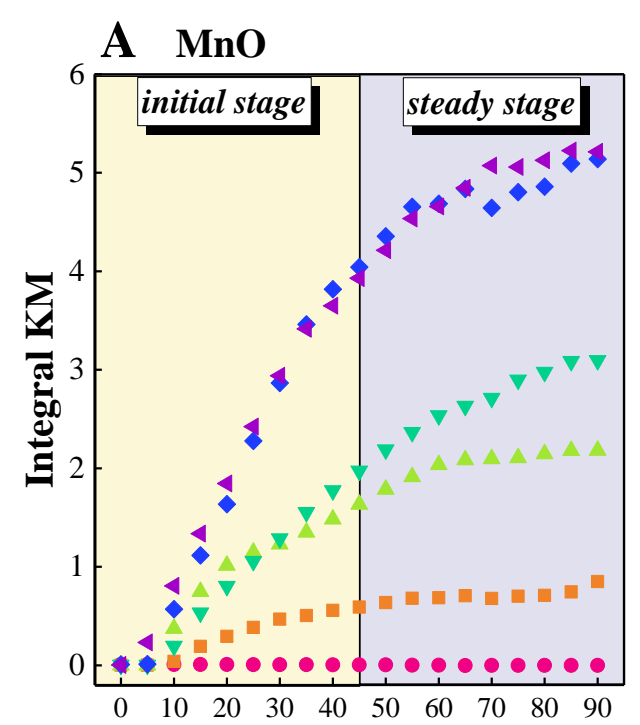

Reaction time (min)

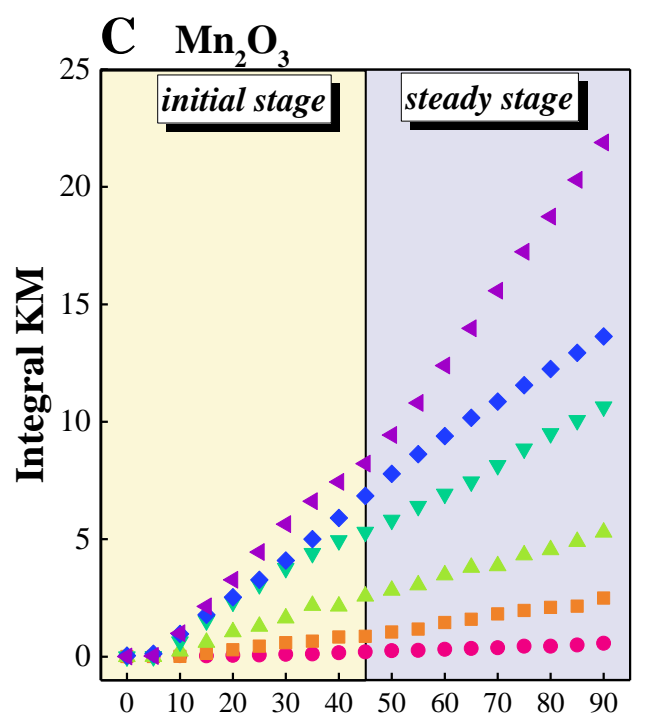

Reaction time (min)

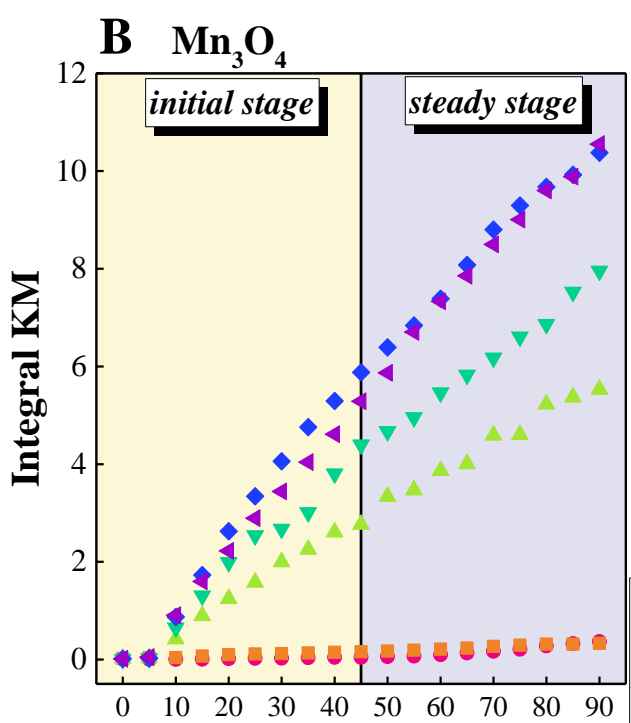

Reaction time (min)

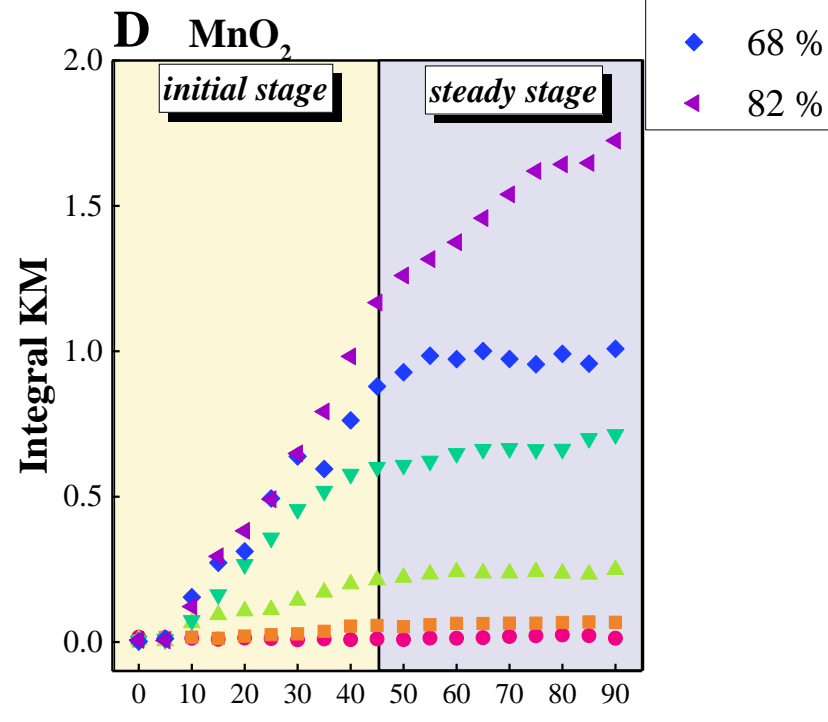

Reaction time (min)

Figure S3. Integral areas for the surface adsorbed water varying with particle type and moisture condition.

The whole heterogeneous process can be divided into initial stage and steady stage according to the accumulation rate of particle surface water. 
Table S2. $\gamma_{\mathrm{BET}}$ values $(\mathrm{Mean} \pm \mathrm{Std})$ for the heterogeneous reaction of $\mathrm{SO}_{2}$ on manganese oxides varying with relative humidity and reaction stage.

\begin{tabular}{|c|c|c|c|c|c|}
\hline & $\mathrm{RH}(\%)$ & $\mathrm{MnO}$ & $\mathrm{Mn}_{3} \mathrm{O}_{4}$ & $\mathrm{Mn}_{2} \mathrm{O}_{3}$ & $\mathrm{MnO}_{2}$ \\
\hline & 0 & $8.02 \times 10^{-8} \pm 1.13 \times 10^{-8}$ & $4.50 \times 10^{-7} \pm 6.86 \times 10^{-8}$ & $4.13 \times 10^{-7} \pm 9.75 \times 10^{-8}$ & $1.84 \times 10^{-7} \pm 7.57 \times 10^{-8}$ \\
\hline & 15 & $6.25 \times 10^{-7} \pm 5.15 \times 10^{-8}$ & $3.33 \times 10^{-7} \pm 3.25 \times 10^{-8}$ & $3.91 \times 10^{-7} \pm 2.74 \times 10^{-8}$ & $4.92 \times 10^{-7} \pm 1.38 \times 10^{-7}$ \\
\hline Initial & 33 & $1.05 \times 10^{-6} \pm 1.18 \times 10^{-7}$ & $1.38 \times 10^{-6} \pm 1.45 \times 10^{-7}$ & $7.29 \times 10^{-7} \pm 6.86 \times 10^{-8}$ & $1.04 \times 10^{-6} \pm 1.46 \times 10^{-7}$ \\
\hline \multirow[t]{6}{*}{ stage } & 50 & $1.21 \times 10^{-6} \pm 1.24 \times 10^{-7}$ & $1.66 \times 10^{-6} \pm 1.52 \times 10^{-7}$ & $1.27 \times 10^{-6} \pm 1.17 \times 10^{-7}$ & $1.72 \times 10^{-6} \pm 2.70 \times 10^{-7}$ \\
\hline & 68 & $1.53 \times 10^{-6} \pm 2.75 \times 10^{-7}$ & $2.27 \times 10^{-6} \pm 2.78 \times 10^{-7}$ & $1.67 \times 10^{-6} \pm 1.66 \times 10^{-7}$ & $2.38 \times 10^{-6} \pm 4.85 \times 10^{-7}$ \\
\hline & 82 & $1.61 \times 10^{-6} \pm 2.23 \times 10^{-7}$ & $1.83 \times 10^{-6} \pm 1.87 \times 10^{-7}$ & $1.11 \times 10^{-6} \pm 1.22 \times 10^{-7}$ & $1.69 \times 10^{-6} \pm 3.42 \times 10^{-7}$ \\
\hline & $\mathrm{RH}(\%)$ & $\mathrm{MnO}$ & $\mathrm{Mn}_{3} \mathrm{O}_{4}$ & $\mathrm{Mn}_{2} \mathrm{O}_{3}$ & $\mathrm{MnO}_{2}$ \\
\hline & 0 & $8.36 \times 10^{-8} \pm 1.11 \times 10^{-8}$ & $2.91 \times 10^{-7} \pm 3.44 \times 10^{-8}$ & $6.80 \times 10^{-7} \pm 1.61 \times 10^{-7}$ & $3.98 \times 10^{-8} \pm 3.81 \times 10^{-8}$ \\
\hline & 15 & $4.24 \times 10^{-7} \pm 7.59 \times 10^{-8}$ & $4.58 \times 10^{-7} \pm 6.19 \times 10^{-8}$ & $1.17 \times 10^{-6} \pm 1.66 \times 10^{-7}$ & $4.41 \times 10^{-7} \pm 1.56 \times 10^{-7}$ \\
\hline \multirow{4}{*}{$\begin{array}{l}\text { Steady } \\
\text { stage }\end{array}$} & 33 & $8.80 \times 10^{-7} \pm 7.78 \times 10^{-8}$ & $3.09 \times 10^{-6} \pm 3.78 \times 10^{-7}$ & $1.73 \times 10^{-6} \pm 1.19 \times 10^{-7}$ & $8.51 \times 10^{-7} \pm 1.19 \times 10^{-7}$ \\
\hline & 50 & $1.39 \times 10^{-6} \pm 2.14 \times 10^{-7}$ & $3.07 \times 10^{-6} \pm 4.12 \times 10^{-7}$ & $2.01 \times 10^{-6} \pm 1.66 \times 10^{-7}$ & $2.30 \times 10^{-6} \pm 3.70 \times 10^{-7}$ \\
\hline & 68 & $1.77 \times 10^{-6} \pm 1.93 \times 10^{-7}$ & $2.98 \times 10^{-6} \pm 2.75 \times 10^{-7}$ & $2.22 \times 10^{-6} \pm 1.52 \times 10^{-7}$ & $2.64 \times 10^{-6} \pm 1.10 \times 10^{-7}$ \\
\hline & 82 & $1.16 \times 10^{-6} \pm 1.06 \times 10^{-7}$ & $2.12 \times 10^{-6} \pm 2.10 \times 10^{-7}$ & $1.94 \times 10^{-6} \pm 2.81 \times 10^{-7}$ & $2.80 \times 10^{-6} \pm 7.40 \times 10^{-7}$ \\
\hline
\end{tabular}


Table S3. Reactive uptake coefficients for the heterogeneous reaction of $\mathrm{SO}_{2}$ on diverse model particles.

\begin{tabular}{|c|c|c|c|c|c|c|}
\hline Particle & Coexisted & Moisture (RH) & Temperature & Illumination & $\gamma$-value & Reference \\
\hline $\mathrm{CaCO}_{3}$ & $\mathrm{O}_{3}$ & $40 \%$ & $293 \mathrm{~K}$ & Dark & $(1.4 \pm 0.3) \times 10^{-7}$ & 1 \\
\hline \multirow{9}{*}{$\mathrm{CaCO}_{3}$} & \multirow{9}{*}{$\mathrm{O}_{3}$} & \multirow{9}{*}{ Dry } & $298 \mathrm{~K}$ & \multirow{9}{*}{ Dark } & $(1.27 \pm 0.13) \times 10^{-7}$ & \multirow{9}{*}{2} \\
\hline & & & $283 \mathrm{~K}$ & & $(1.28 \pm 0.10) \times 10^{-7}$ & \\
\hline & & & $273 \mathrm{~K}$ & & $(1.56 \pm 0.09) \times 10^{-7}$ & \\
\hline & & & $263 \mathrm{~K}$ & & $(1.73 \pm 0.05) \times 10^{-7}$ & \\
\hline & & & $257 \mathrm{~K}$ & & $(2.19 \pm 0.19) \times 10^{-7}$ & \\
\hline & & & $250 \mathrm{~K}$ & & $(2.36 \pm 0.07) \times 10^{-7}$ & \\
\hline & & & $245 \mathrm{~K}$ & & $(2.37 \pm 0.28) \times 10^{-7}$ & \\
\hline & & & $240 \mathrm{~K}$ & & $(2.08 \pm 0.28) \times 10^{-7}$ & \\
\hline & & & $230 \mathrm{~K}$ & & $(1.53 \pm 0.13) \times 10^{-7}$ & \\
\hline \multirow{10}{*}{$\mathrm{CaCO}_{3}$} & \multirow{10}{*}{$\mathrm{O}_{3}$} & $1 \%$ & \multirow{10}{*}{$298 \mathrm{~K}$} & \multirow{10}{*}{ Dark } & $(2.59 \pm 0.17) \times 10^{-7}$ & \multirow{10}{*}{3} \\
\hline & & $18 \%$ & & & $(3.02 \pm 0.05) \times 10^{-7}$ & \\
\hline & & $29 \%$ & & & $(3.46 \pm 0.04) \times 10^{-7}$ & \\
\hline & & $40 \%$ & & & $(3.98 \pm 0.04) \times 10^{-7}$ & \\
\hline & & $52 \%$ & & & $(3.84 \pm 0.14) \times 10^{-7}$ & \\
\hline & & $65 \%$ & & & $(3.99 \pm 0.09) \times 10^{-7}$ & \\
\hline & & $74 \%$ & & & $(3.87 \pm 0.22) \times 10^{-7}$ & \\
\hline & & $80 \%$ & & & $(3.84 \pm 0.16) \times 10^{-7}$ & \\
\hline & & $85 \%$ & & & $(3.87 \pm 0.04) \times 10^{-7}$ & \\
\hline & & $90 \%$ & & & $(3.90 \pm 0.01) \times 10^{-7}$ & \\
\hline
\end{tabular}

Page S7 


\begin{tabular}{|c|c|c|c|c|c|c|}
\hline $\mathrm{TiO}_{2}$ & -- & $\begin{array}{r}0 \% \\
40 \% \\
\end{array}$ & $298 \mathrm{~K}$ & $\begin{array}{c}\text { Dark } \\
\text { Ultraviolet }\end{array}$ & $\begin{array}{l}1.94 \times 10^{-6} \\
1.35 \times 10^{-5} \\
\end{array}$ & 4 \\
\hline \multirow{8}{*}{$\mathrm{TiO}_{2}$} & \multirow{4}{*}{$\mathrm{O}_{3}$} & $4 \%$ & \multirow{8}{*}{$298 \mathrm{~K}$} & \multirow{8}{*}{ Dark } & $(1.42 \pm 0.12) \times 10^{-7}$ & \multirow{8}{*}{5} \\
\hline & & $35 \%$ & & & $\sim 3 \times 10^{-7}$ & \\
\hline & & $58 \%$ & & & $\sim 4 \times 10^{-7}$ & \\
\hline & & $85 \%$ & & & $(6.23 \pm 0.50) \times 10^{-7}$ & \\
\hline & \multirow{4}{*}{$\mathrm{NO}_{2}$} & $3 \%$ & & & $(1.01 \pm 0.09) \times 10^{-7}$ & \\
\hline & & $32 \%$ & & & $\sim 2 \times 10^{-7}$ & \\
\hline & & $56 \%$ & & & $\sim 3 \times 10^{-7}$ & \\
\hline & & $84 \%$ & & & $(4.83 \pm 0.43) \times 10^{-7}$ & \\
\hline \multirow{9}{*}{$\alpha-\mathrm{Al}_{2} \mathrm{O}_{3}$} & \multirow{4}{*}{$\mathrm{O}_{3}$} & $15 \%$ & \multirow{9}{*}{$298 \mathrm{~K}$} & \multirow{9}{*}{ Dark } & $(3.66 \pm 0.91) \times 10^{-7}$ & \multirow{9}{*}{6} \\
\hline & & $34 \%$ & & & $(1.36 \pm 0.41) \times 10^{-6}$ & \\
\hline & & $54 \%$ & & & $(1.96 \pm 1.02) \times 10^{-6}$ & \\
\hline & & $95 \%$ & & & $(2.42 \pm 0.77) \times 10^{-6}$ & \\
\hline & \multirow{5}{*}{$\mathrm{NO}_{2}$} & $15 \%$ & & & $(0.42 \pm 0.17) \times 10^{-7}$ & \\
\hline & & $30 \%$ & & & $(1.54 \pm 0.46) \times 10^{-7}$ & \\
\hline & & $50 \%$ & & & $(2.96 \pm 1.18) \times 10^{-7}$ & \\
\hline & & $69 \%$ & & & $(5.64 \pm 1.69) \times 10^{-7}$ & \\
\hline & & $90 \%$ & & & $(5.95 \pm 2.98) \times 10^{-7}$ & \\
\hline \multirow{4}{*}{ Soot } & \multirow{4}{*}{$\mathrm{O}_{3}$} & $1 \%$ & \multirow{4}{*}{$298 \mathrm{~K}$} & \multirow{4}{*}{ Dark } & $(0.81 \pm 0.07) \times 10^{-6}$ & \multirow{4}{*}{7} \\
\hline & & $31 \%$ & & & $(1.82 \pm 0.09) \times 10^{-6}$ & \\
\hline & & $52 \%$ & & & $(2.97 \pm 0.15) \times 10^{-6}$ & \\
\hline & & $83 \%$ & & & $(9.95 \pm 0.31) \times 10^{-6}$ & \\
\hline
\end{tabular}

Page S8 

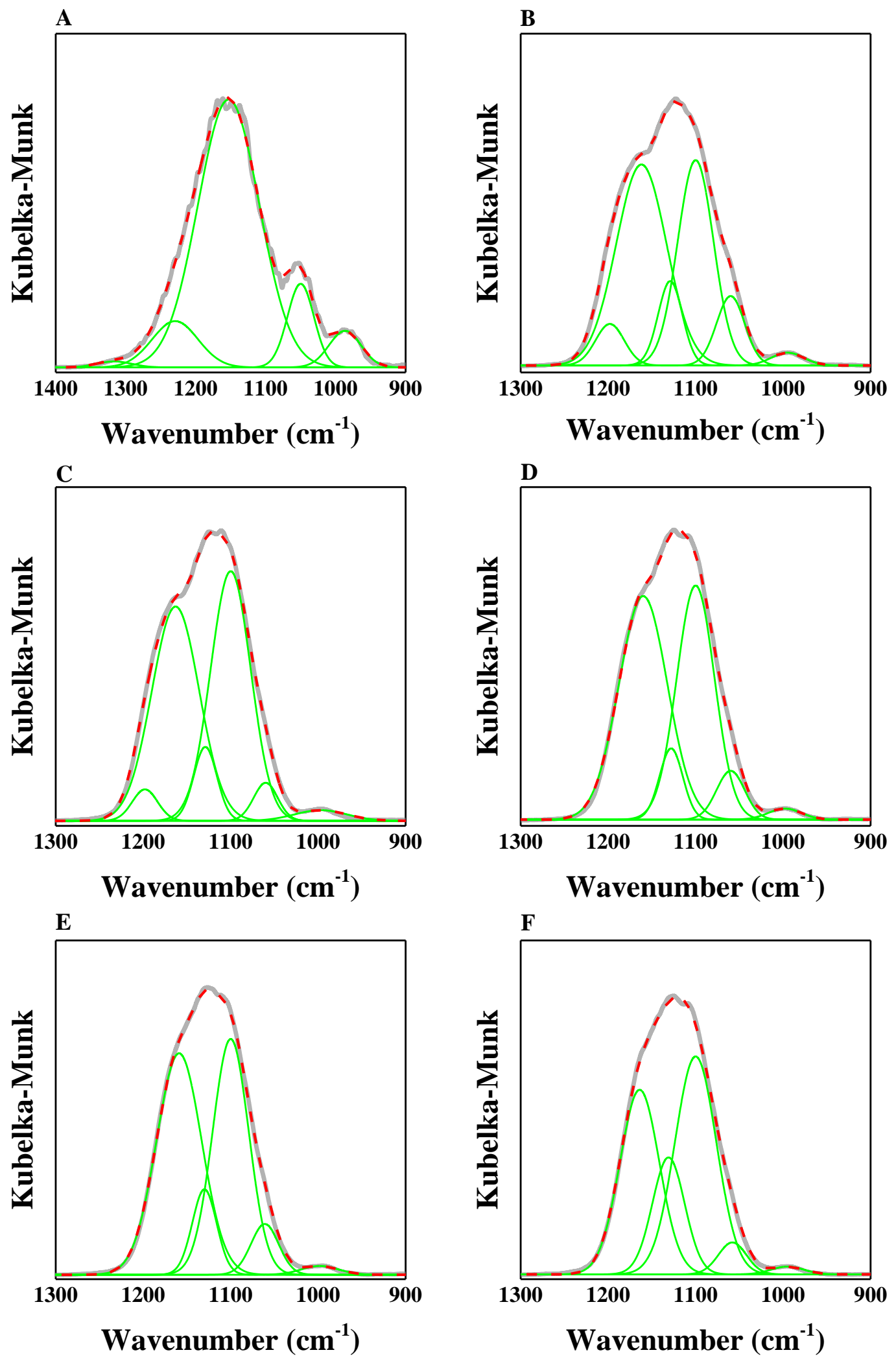

Figure S4. Curve-fitting of the last DRIFTS spectra for $\mathrm{MnO}$ under various RHs. (A-F: $\mathrm{RH}=0 \%, 15 \%$, $33 \%, 50 \%, 68 \%$, and $82 \%$, respectively) 

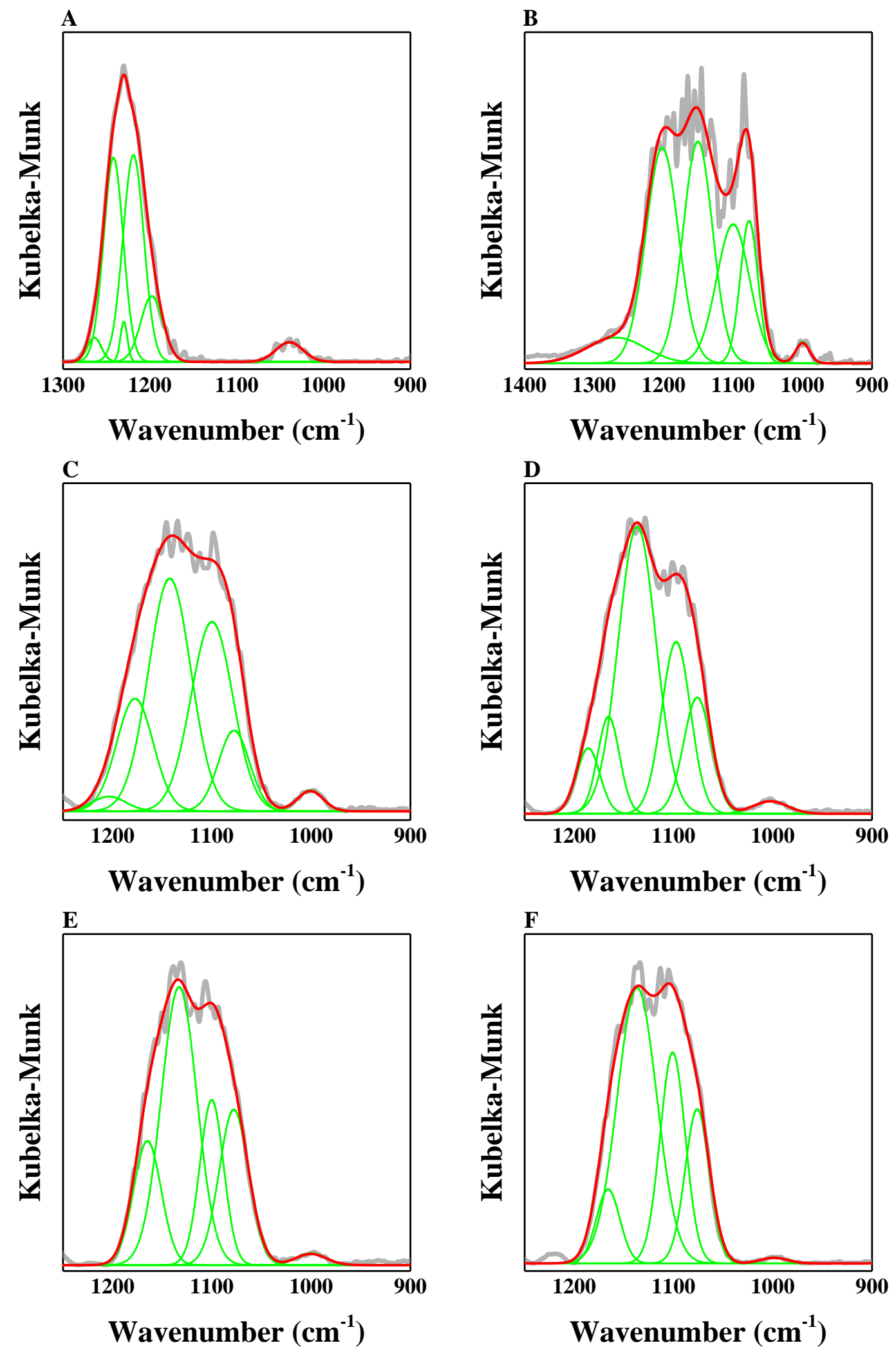

Figure S5. Curve-fitting of the last DRIFTS spectra for $\mathrm{Mn}_{3} \mathrm{O}_{4}$ under various RHs. (A-F: $\mathrm{RH}=0 \%, 15 \%$, $33 \%, 50 \%, 68 \%$, and $82 \%$, respectively) 

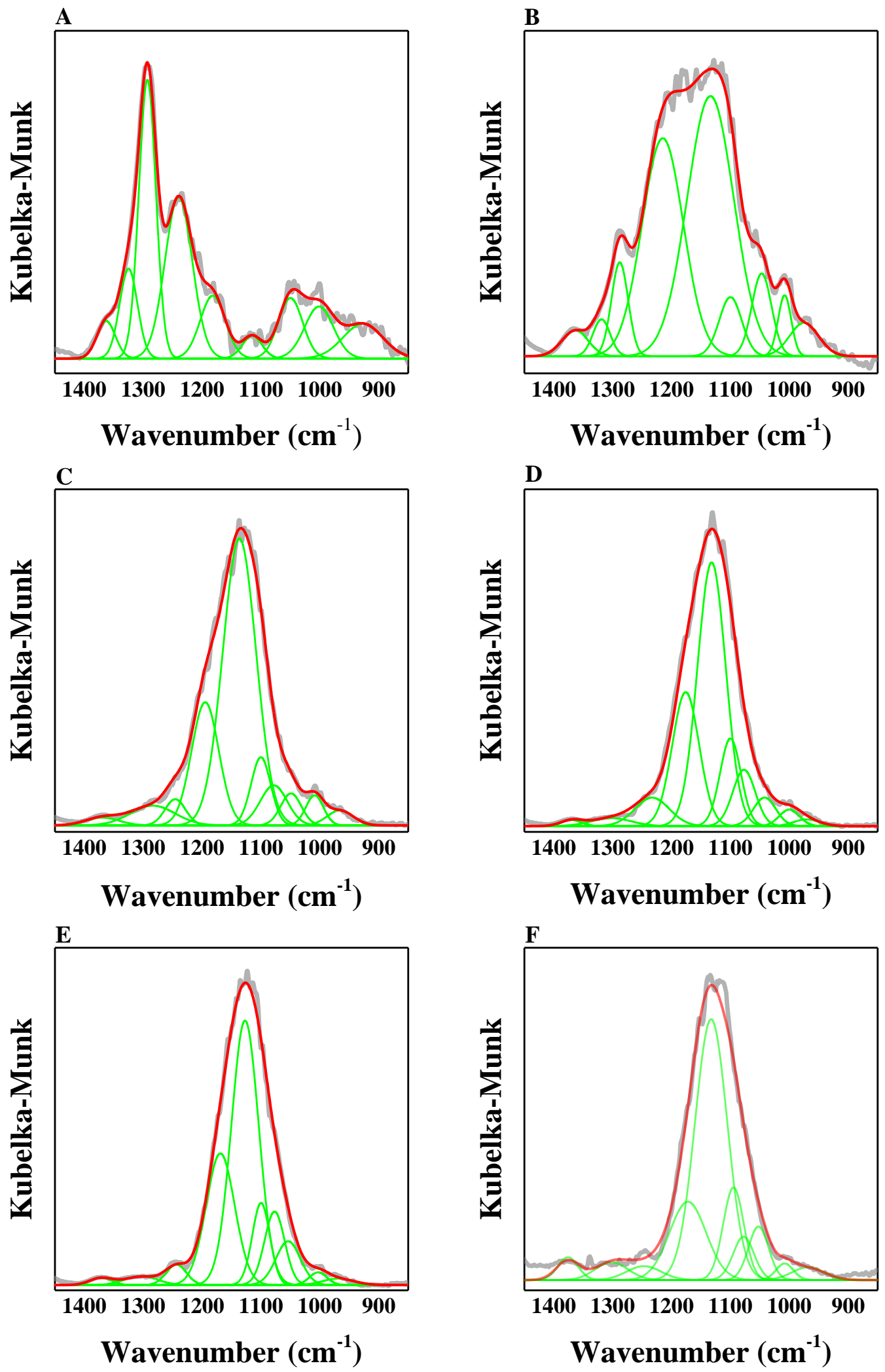

Figure S6. Curve-fitting of the last DRIFTS spectra for $\mathrm{Mn}_{2} \mathrm{O}_{3}$ under various RHs. (A-F: $\mathrm{RH}=0 \%, 15 \%$, $33 \%, 50 \%, 68 \%$, and $82 \%$, respectively) 

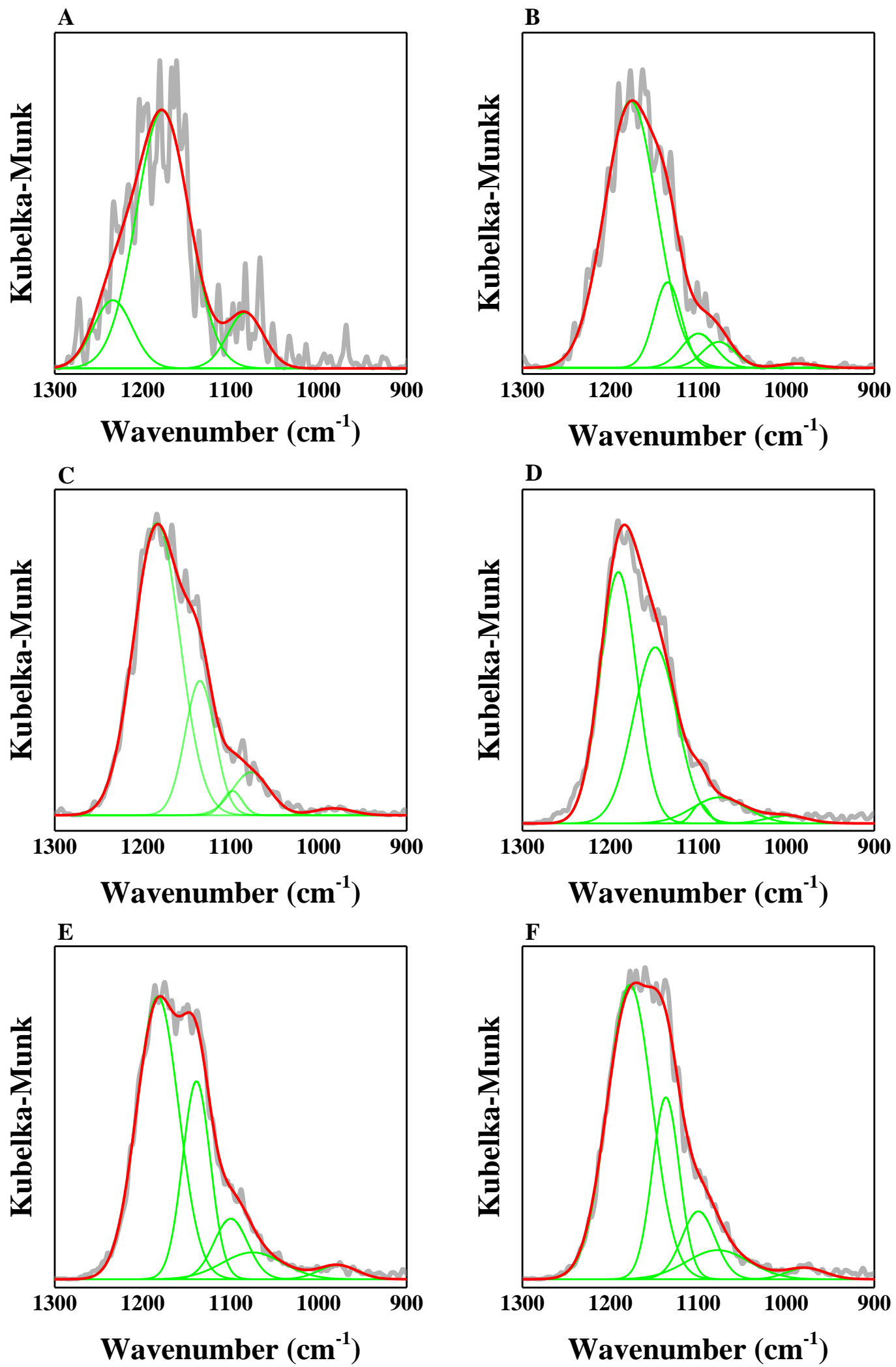

Figure S7. Curve-fitting of the last DRIFTS spectra for $\mathrm{MnO}_{2}$ under various RHs. (A-F: $\mathrm{RH}=0 \%, 15 \%$, $33 \%, 50 \%, 68 \%$, and $82 \%$, respectively) 


\section{References}

(1) Li, L.; Chen, Z. M.; Zhang, Y. H.; Zhu, T.; Li, J. L.; Ding, J. Kinetics and mechanism of heterogeneous oxidation of sulfur dioxide by ozone on surface of calcium carbonate. Atmos. Chem. Phys. 2006, 6, 2453-2464.

(2) Wu, L. W.; Tong, S. R.; Wang, W. G.; Ge, M. F. Effects of temperature on the heterogeneous oxidation of sulfur dioxide by ozone on calcium carbonate. Atmos. Chem. Phys. 2011, 11, 6593-6605.

(3) Zhang, Y.; Tong, S.; Ge, M.; Jing, B.; Hou, S.; Tan, F.; Chen, Y.; Guo, Y.; Wu, L. The influence of relative humidity on the heterogeneous oxidation of sulfur dioxide by ozone on calcium carbonate particles. Sci. Total Environ. 2018, 633, 1253-1262.

(4) Shang, J.; Li, J.; Zhu, T. Heterogeneous reaction of $\mathrm{SO}_{2}$ on $\mathrm{TiO}_{2}$ particles. Science China Chemistry. 2010, 53, 2637-2643.

(5) He, X.; Zhang, Y. Influence of relative humidity on $\mathrm{SO}_{2}$ oxidation by $\mathrm{O}_{3}$ and $\mathrm{NO}_{2}$ on the surface of $\mathrm{TiO}_{2}$ particles: Potential for formation of secondary sulfate aerosol. Spectrochimica Acta Part A: Molecular and Biomolecular Spectroscopy. 2019, 219, 121-128.

(6) Liu, W.; He, X.; Pang, S.; Zhang, Y. Effect of relative humidity on $\mathrm{O}_{3}$ and $\mathrm{NO}_{2}$ oxidation of $\mathrm{SO}_{2}$ on $\alpha-\mathrm{Al}_{2} \mathrm{O}_{3}$ particles. Atmos. Environ. 2017, 167, 245-253.

(7) He, X.; Pang, S.; Ma, J.; Zhang, Y. Influence of relative humidity on heterogeneous reactions of $\mathrm{O}_{3}$ and $\mathrm{O}_{3} / \mathrm{SO}_{2}$ with soot particles: Potential for environmental and health effects. Atmos. Environ. 2017, 165, 198-206. 